\section{Original Article}

Basic Medical Sciences

Check for updates

\title{
Advanced Sectioned Images of a Cadaver Head with Voxel Size of $0.04 \mathrm{~mm}$
}

\author{
Beom Sun Chung $\left(\mathbb{D},{ }^{1}\right.$ Miran Han $\mathbb{D}^{1},{ }^{2}$ Donghwan Har $\mathbb{D}^{1},{ }^{3}$ and Jin Seo Park $(\mathbb{D})^{4}$ \\ 'Department of Anatomy, Ajou University School of Medicine, Suwon, Korea \\ ${ }^{2}$ Department of Radiology, Ajou University School of Medicine, Suwon, Korea \\ ${ }^{3}$ College of ICT Engineering, Chung Ang University, Seoul, Korea \\ ${ }^{4}$ Department of Anatomy, Dongguk University School of Medicine, Gyeongju, Korea
}

Received: Jun 14, 2019

Accepted: Jul 22, 2019

Address for Correspondence:

Jin Seo Park, PhD

Department of Anatomy, Dongguk University School of Medicine, 87 Dongdae-ro, Gyeongju 38067, Republic of Korea.

E-mail: park93@dongguk.ac.kr

(c) 2019 The Korean Academy of Medical Sciences.

This is an Open Access article distributed under the terms of the Creative Commons Attribution Non-Commercial License (https:// creativecommons.org/licenses/by-nc/4.0/) which permits unrestricted non-commercial use, distribution, and reproduction in any medium, provided the original work is properly cited.

ORCID IDs

Beom Sun Chung (D)

https://orcid.org/0000-0002-3644-9120 Miran Han (1)

https://orcid.org/0000-0001-7752-5858

Donghwan Har (D)

https://orcid.org/0000-0002-8676-8242 Jin Seo Park (iD)

https://orcid.org/0000-0001-7956-4148

Funding

This research was financially supported by the Ministry of Trade, Industry and Energy (MOTIE) and Korea Institute for Advancement of Technology (KIAT) through the International Cooperative R\&D program (Grant No. Noo02249).

\section{ABSTRACT}

Background: The sectioned images of a cadaver head made from the Visible Korean project have been used for research and educational purposes. However, the image resolution is insufficient to observe detailed structures suitable for experts. In this study, advanced sectioned images with higher resolution were produced for the identification of more detailed structures.

Methods: The head of a donated female cadaver was scanned for 3 Tesla magnetic resonance images and diffusion tensor images (DTIs). After the head was frozen, the head was sectioned serially at $0.04-\mathrm{mm}$ intervals and photographed repeatedly using a digital camera. Results: On the resulting 4,000 sectioned images (intervals and pixel size, $0.04 \mathrm{~mm}^{3}$; color depth, 48 bits color; a file size, 288 Mbytes), minute brain structures, which can be observed not on previous sectioned images but on microscopic slides, were observed. The voxel size of this study $\left(0.04 \mathrm{~mm}^{3}\right)$ was very minute compared to our previous study $\left(0.1 \mathrm{~mm}^{3}\right.$; resolution, $4,368 \times 2,912)$ and Visible Human Project of the USA $\left(0.33 \mathrm{~mm}^{3}\right.$; resolution, 2,048 $\left.\times 2,048\right)$. Furthermore, the sectioned images were combined with tractography of the DTIs to elucidate the white matter with high resolution and the actual color of the tissue.

Conclusion: The sectioned images will be used for diverse research, including the applications for the cross sectional anatomy and three-dimensional models for virtual experiments.

Keywords: Cadaver; Cross-Sectional Anatomy; Diffusion Tensor Imaging; Head; Visible Human Project

\section{INTRODUCTION}

In 2009, the sectioned images of a cadaver head were produced as a part of the Visible Korean. ${ }^{1}$ Owing to the real color (48 bits color) and small voxel size $(0.1 \mathrm{~mm} \times 0.1 \mathrm{~mm} \times 0.1$ $\mathrm{mm}$ ) of the images, various outcomes for medical education and research were produced. Neuroanatomy atlases, consisting of only sectioned images ${ }^{2}$ and sectioned images with 7 Tesla (T) magnetic resonance images (MRIs), ${ }^{3}$ have been published. Using the sectioned images and segmented data, two dimensional applications for personal computers 4,5 and android mobile devices 6 have been produced for widespread use. Moreover, surface and volume models of diverse head structures have been produced.-12 Currently, real color 
Disclosure

The authors have no potential conflicts of interest to disclose.

Author Contributions

Conceptualization: Park JS. Data curation: Han

M, Har D. Formal analysis: Park JS, Chung BS.

Investigation: Park JS. Methodology: Park JS,

Har D. Software: Chung BS. Validation: Park

JS. Writing - original draft: Chung BS, Park JS.

Writing - review \& editing: Park JS. sectioned images of Visible Korean are essential materials for learning neuroanatomy, sectional anatomy, and radiology and have opened a new chapter of sophisticated gross anatomy research.

On the other hand, the resolution of the images made in 2009 was insufficient to observe detailed structures, such as each nuclei of the thalamus, ${ }^{13}$ deep nuclei in the cerebellum, ${ }^{14}$ brainstem nuclei, ${ }^{15}$ and the connections of each fiber in the brain white matter. ${ }^{16}$ These detailed structures should be identified to provide a morphological background to neuroscience research. ${ }^{17}$ Moreover, the sectioned images in 2009 were not accompanied by 3T MRIs and diffusion tensor images (DTIs) that can be the reference data for interpreting radiologic images. 18

The purpose of this study was to produce advanced sectioned images of the head with a higher resolution than previous sectioned images. In addition, the 3T MRIs and DTIs of the same cadaver were presented together. The advanced images enabled the observation of minute structures that were not visible in the previous images.

\section{METHODS}

\section{Cadaver preparation and medical imaging}

A female cadaver without a head pathology was donated. For a further alignment process, three radiologic markers were attached to the midline of the anterior side of the head, while two of them were attached to the posterior side. Within 24 hours after death, the head region of the cadaver was scanned for 3T MRIs using both T1 and T2 methods (repetition time, 2,500 ms; echo time, $8 \mathrm{~ms}$ ). While scanning the MRIs, the reference lines by the anterior commissure (AC) and posterior commissure (PC) were identified to determine the horizontal, coronal, and sagittal planes. ${ }^{19}$ In addition, the DTIs were also scanned (Table 1 and Fig. 1 ).

The MRIs were volume reconstructed using MRIcroGL software. ${ }^{20}$ By observing the horizontal, coronal, and sagittal planes on the volume model, the reference lines for the alignment could be drawn on the skin according to the radiologic markers. The cadaver was frozen in a deep freezer

Table 1. Specification comparison between data of previous study and data of this study

\begin{tabular}{lcc}
\hline Variables & Data of previous study26 & Data of this study \\
\hline Sex & Male & Female \\
Age at death & 67-year-old & 70-year-old \\
Cause of death & Myasthenia gravis & Guillain-Barré syndrome \\
MRIs & T1 and T2 of 1.5 Tesla & T1 and T2 of 3 Tesla \\
DTIs & Not scanned & Scanned \\
CTs & Scanned & Not scanned \\
Sectioned images & & \\
Digital camera & Canon EOS 5D (released in 2005) & Canon EOS 5Ds R (released in 2015) \\
Color depth & 48 bits color & 48 bits color \\
Resolution & $4,368 \times 2,912$ & $8,688 \times 5,792$ \\
Interval & $0.1 \mathrm{~mm}$ & 0.04 mm \\
Pixel size & $0.1 \mathrm{~mm} \times 0.1 \mathrm{~mm}$ & 0.04 mm $\times 0.04 \mathrm{~mm}$ \\
Number of images & 2,343 & 4,000 \\
File format & TIFF & TIFF \\
File size of an image & $72 \mathrm{MBytes}$ & $288 \mathrm{MBytes}$ \\
Total file size & $166 \mathrm{GBytes}$ & $1,090 \mathrm{GBytes}$ \\
\hline
\end{tabular}

$\mathrm{MRI}=$ magnetic resonance images, $\mathrm{DTI}=$ diffusion tensor image, CT = computerized tomography, $\mathrm{TIFF}=\mathrm{tagged}$ image file format. 


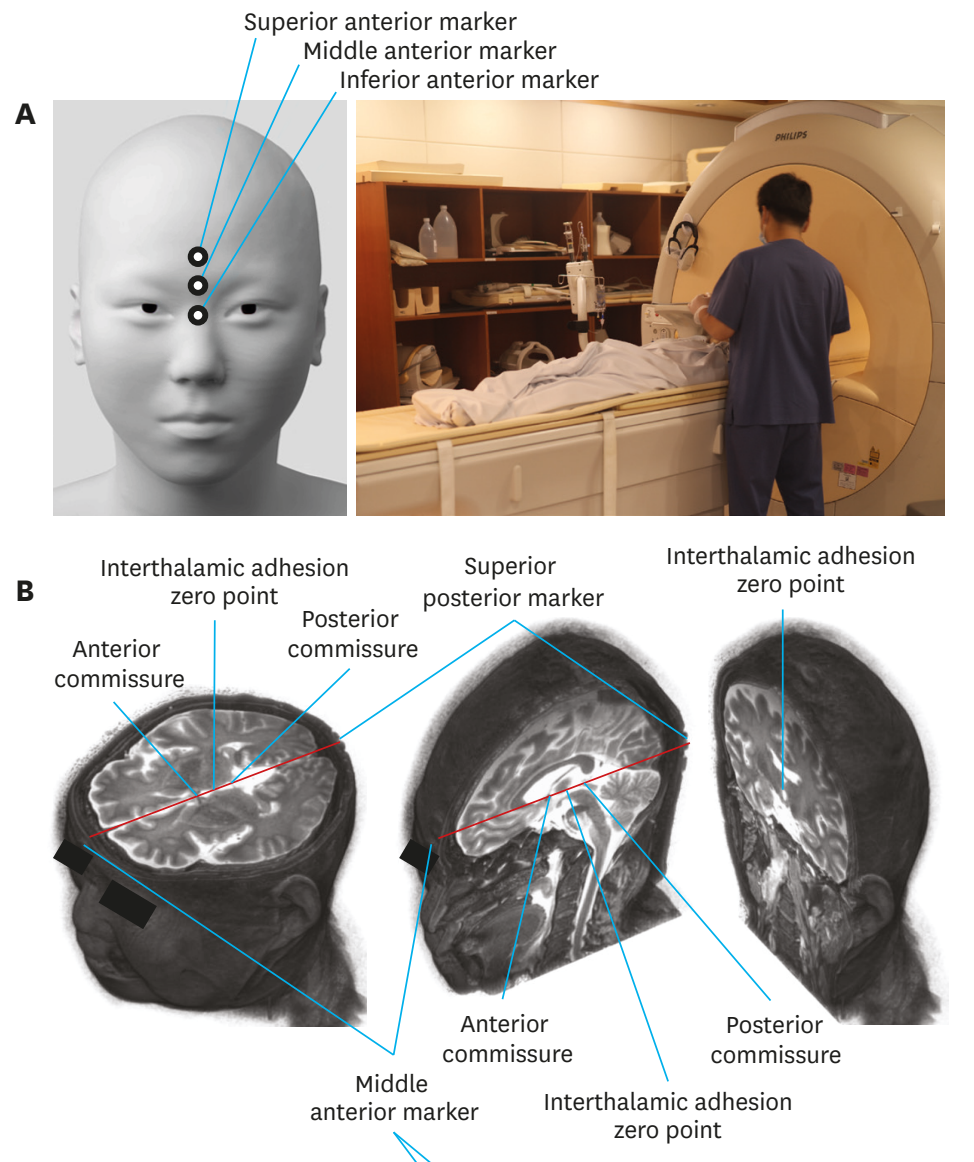

C

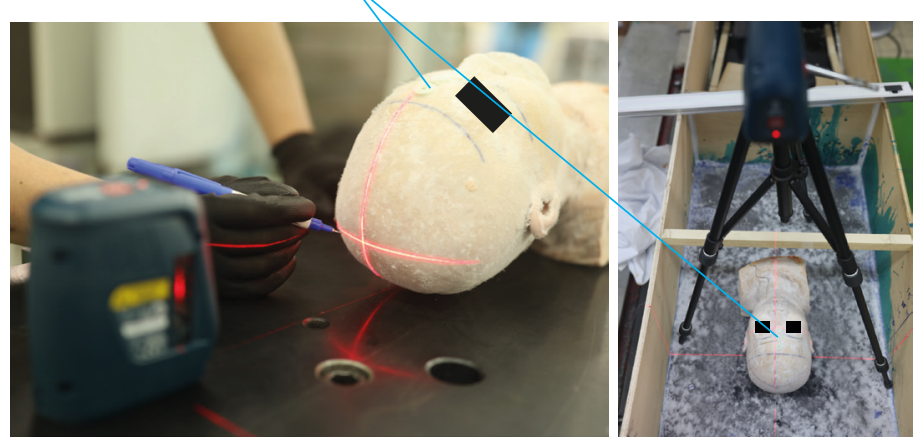

D
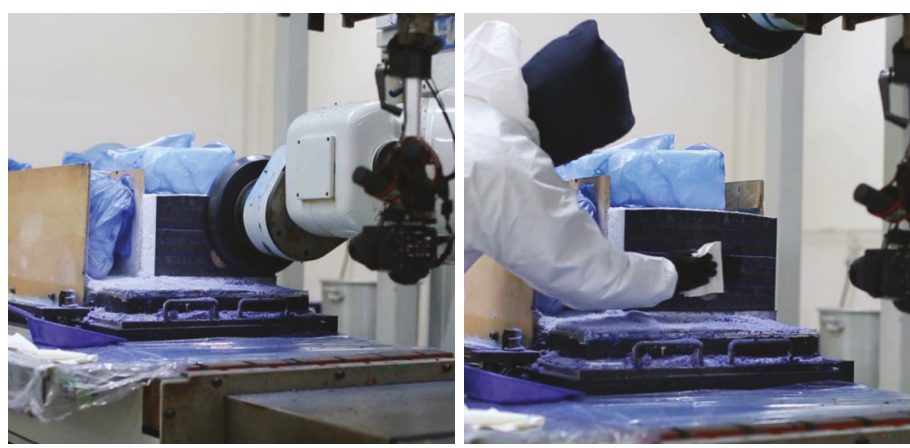

Fig. 1. Overall production process of cadaver images. (A) Cadaveric head with the radiologic markers on skin being scanned for MRIs. (B) The anterior commissure-posterior commissure line indicated as red lines on the horizontal and sagittal planes of the volume model made from the MRIs. (C) Cadaveric head being placed on the embedding box using a cross line laser device for the alignment. (D) Embedding box including the head placed on the cryomacrotome, the head sectioned at 0.04-mm intervals, and frost on the sectioned surface being wiped away. 
at -70 degrees Celsius. After freezing, the cadaver head was detached from the rest of body and placed on an embedding box. To align the head according to the horizontal, coronal, and sagittal planes of the MRIs, the reference lines on the skin were matched to the cross line laser. The placed head was embedded in a gelatin solution and frozen again (Fig. 1).

\section{Serial sectioning}

The frozen cadaveric head was sectioned serially using a cryomacrotome. Sectioning was performed serially at 0.04 -mm intervals. After every sectioning, frost and foreign substances on the sectioned surface were wiped away with 99\% ethyl alcohol. Protruding objects on the surface were removed using a scalpel (Fig. 1).

A Canon ${ }^{\mathrm{TM}}$ EOS 5DsR digital single lens reflex camera and Canon ${ }^{\mathrm{TM}} \mathrm{EF} 100 \mathrm{~mm}$ f/2.8L Macro IS USM Lens were employed (resolution, 8,688 $\times 5,792$ pixels). The distance from the digital camera to the sectioned surface was adjusted to yield a photograph area of $347.5 \mathrm{~mm}$ in horizontal length and $231.7 \mathrm{~mm}$ in vertical length on the sectioned surface, corresponding to a pixel size of $0.04 \mathrm{~mm} \times 0.04 \mathrm{~mm}$. Two Elinchrom ${ }^{\mathrm{TM}}$ Digital S Strobes with an Elinchrom ${ }^{\mathrm{TM}}$ Digital 2 power pack were used to maintain a constant brightness of the sectioned surface. A Tiffen ${ }^{\mathrm{TM}}$ Color Control Patch was placed on the sectioned surface and photographed every day for post processing of these images. The sectioned surface was photographed using a digital camera (ISO 100, shutter speed 1/250, aperture F/13, manual focus). The photograph was assessed by anatomists on a computer monitor using Adobe Photoshop CS6 (Adobe Systems, Inc., San Jose, CA, USA).

\section{Ethics statement}

This study was approved by the Institution Review Board (IRB) of Ajou University School of Medicine (IRB No. AJIRB-MED-MDB-18-315). Regarding the cadaver donation and further processes, informed consent was acquired from the donor and families.

\section{RESULTS}

Four thousand sectioned images of the whole head were produced at $0.04 \mathrm{~mm}$ intervals, $0.04 \mathrm{~mm} \times 0.04 \mathrm{~mm}$ sized-pixel, 48 bits color, and tagged image file format (TIFF). The file size of a single image was 288 Mbytes while that of the total 4,000 images was 1,090 GBytes (Table 1). Furthermore, the sectioned images corresponded to the 3T MRIs (T1, T2) and DTIs of the identical cadaver. Unlike the MRIs, the sectioned images enabled direct observations of the actual color of the inside structures of the cadaver head. The DTIs enabled direct observations of each tract of the white matter (Fig. 2).

Detailed structures of the cerebrum and cerebellum were observed on the sectioned images. As a representative, the thalamus was divided according to the grain of the tissue, which corresponded to the typical taxonomy. ${ }^{13,21}$ For example, the hippocampus on the sectioned images had a similar curvature to that of neurohistological stains. The cellular layers of the lateral geniculate nucleus could be distinguished on the sectioned images without staining. The cerebellar nuclei could be identified on the sectioned images, similar to the neurohistological stains (Fig. 3).22-24

Detailed structures from the brainstem to the spinal cord were observed on the sectioned images. In the midbrain, the oculomotor nucleus and medial longitudinal fasciculus were 
A
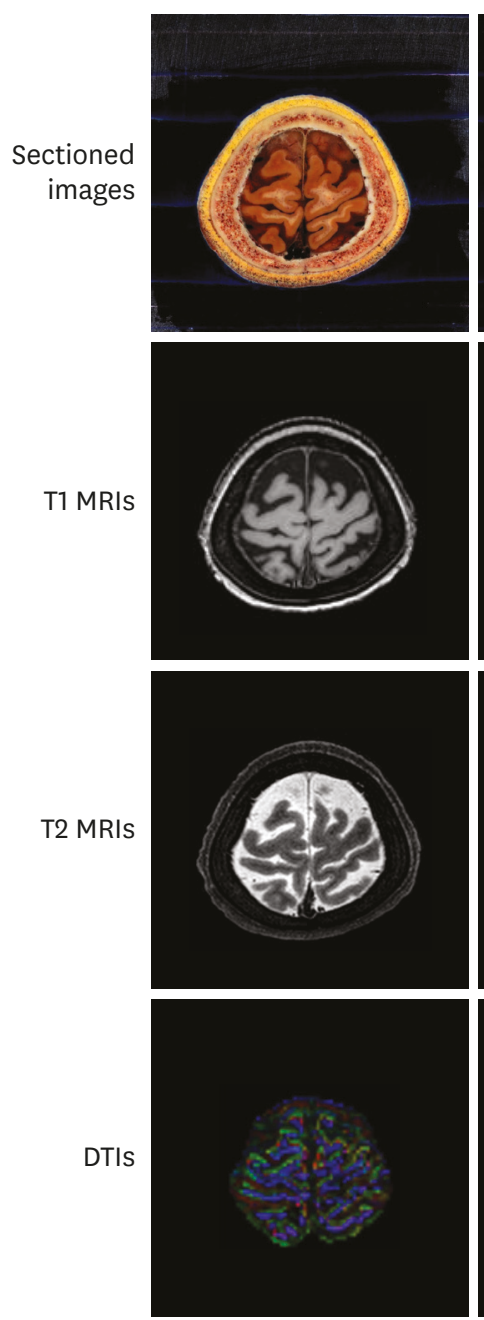

B
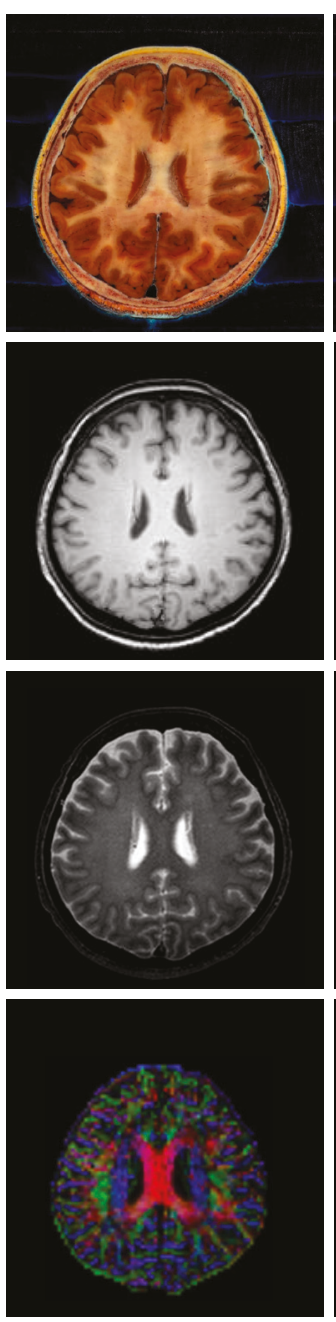

C
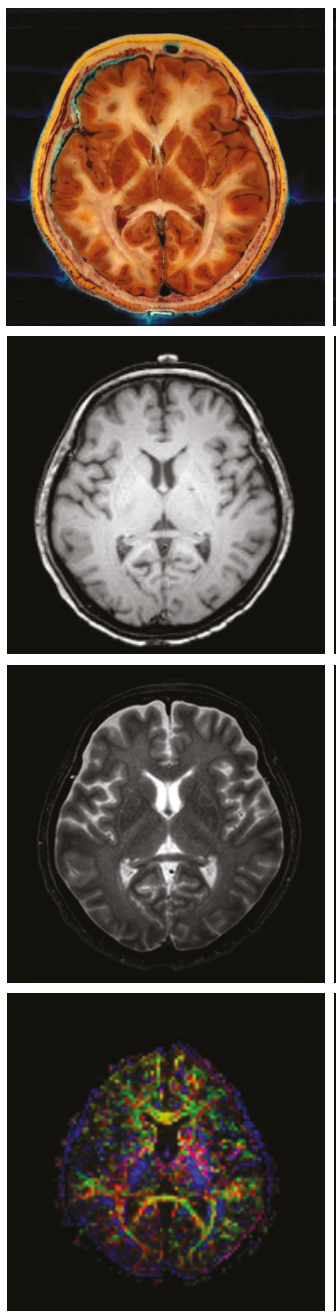

D
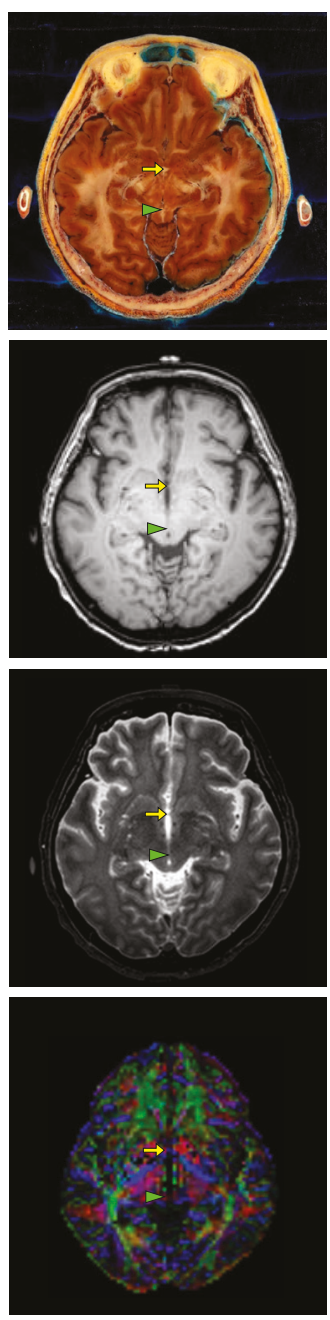

E
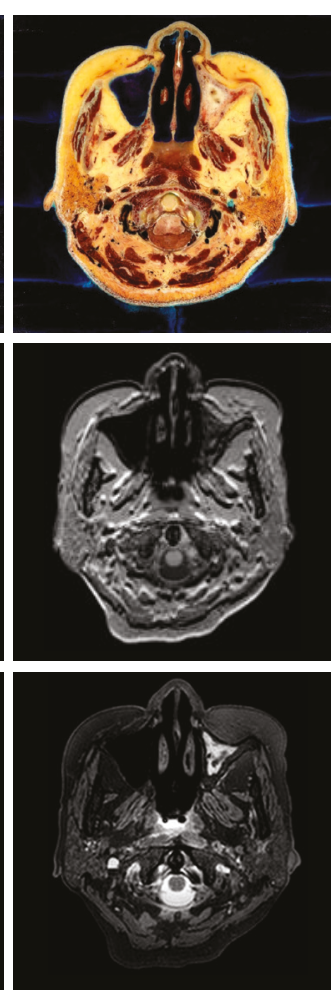

Fig. 2. Sectioned images, T1 MRIs, T2 MRIs, and DTIs on the horizontal planes. Images are at the levels of (A) the crown, (B) body of corpus callosum, (C) lentiform nucleus, (D) horizontal 0-mm plane containing anterior commissure (yellow arrow) and posterior commissure (green arrow head), and (E) neck. $\mathrm{MRI}=$ magnetic resonance image, $\mathrm{T}=\mathrm{Tesla}, \mathrm{DTI}=$ diffusion tensor image .

observed. In the pons, it was possible to observe small arteries, nerve fibers, and nuclei like the superior central nucleus of the raphe and dorsal raphe nucleus. In the medulla oblongata, the nuclei of the cranial nerves were identified, such as the solitary nucleus and hypoglossal nucleus. In the cervical segment of the spinal cord, the lateral corticospinal tract invaded almost the entire area of the posterior horn. The medial longitudinal fasciculus and spinothalamic tract were observed from the brainstem to the spinal cord. The brainstem could be identified on the sectioned images, similar to the neurohistological stains (Fig. 4).

White matter fibers can be observed on a manually dissected brain, and they could also be observed on the sectioned images of this study. For verification, the sectioned image at the level of the thalamus was compared with the 3D tractograph made from the DTIs of the same cadaver. The anterior thalamic radiation and superior thalamic radiation were identified on both the sectioned images and 3D tractography (Fig. 5). 

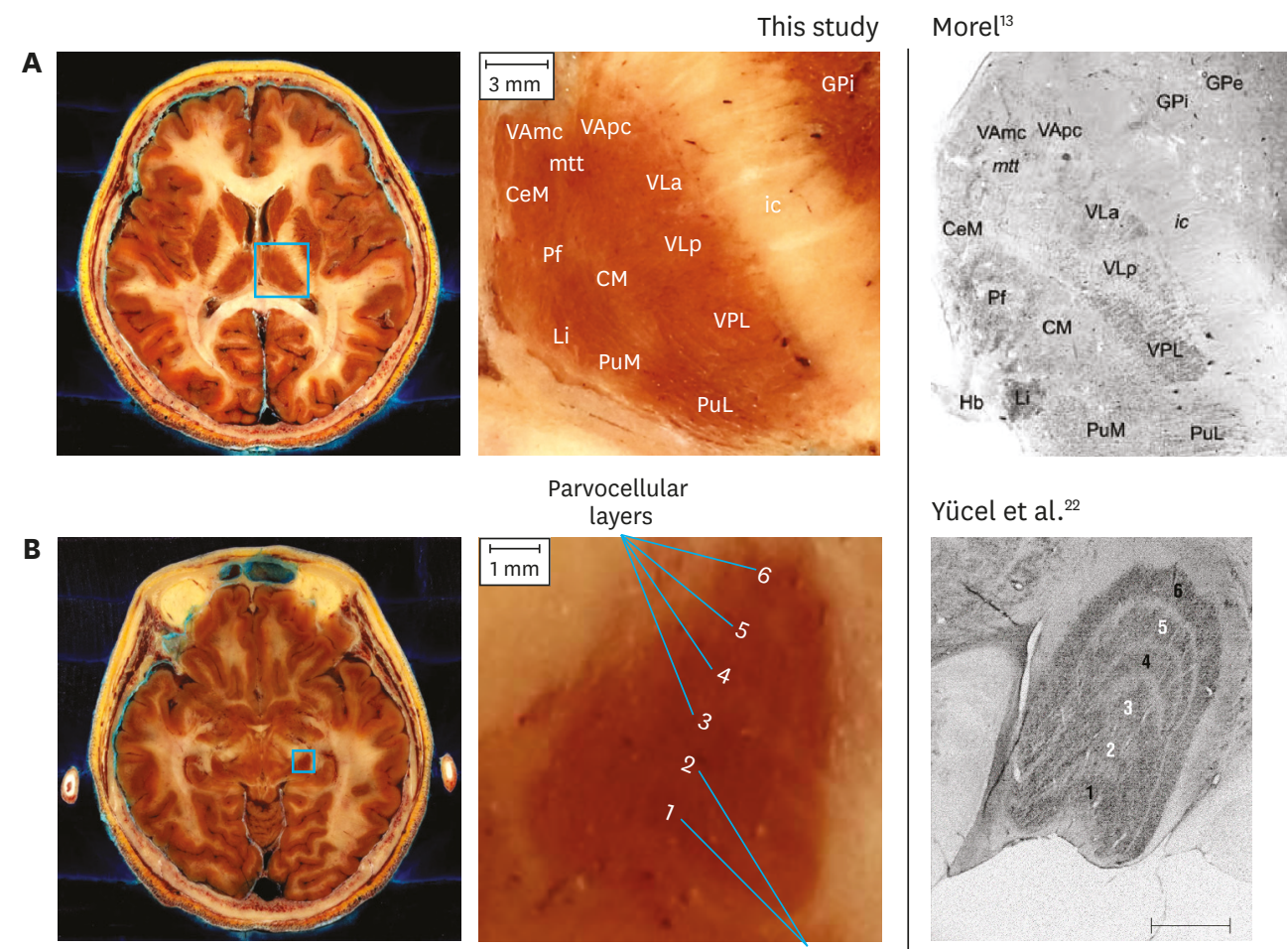

Parvocellular layers

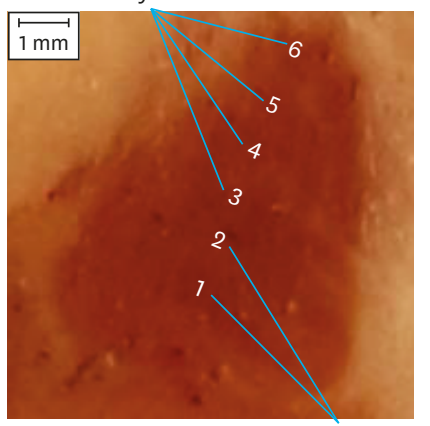

Magnocellular

layers
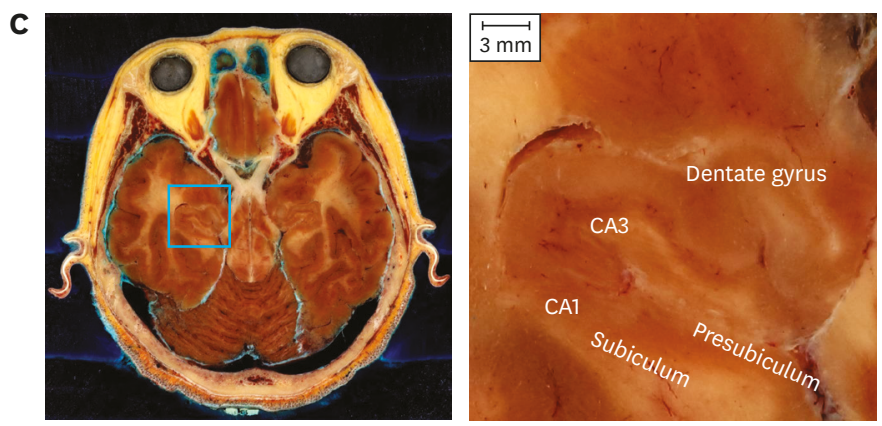

Dorsal dentate Emboliform Fastigial nucleus nucleus nucleus
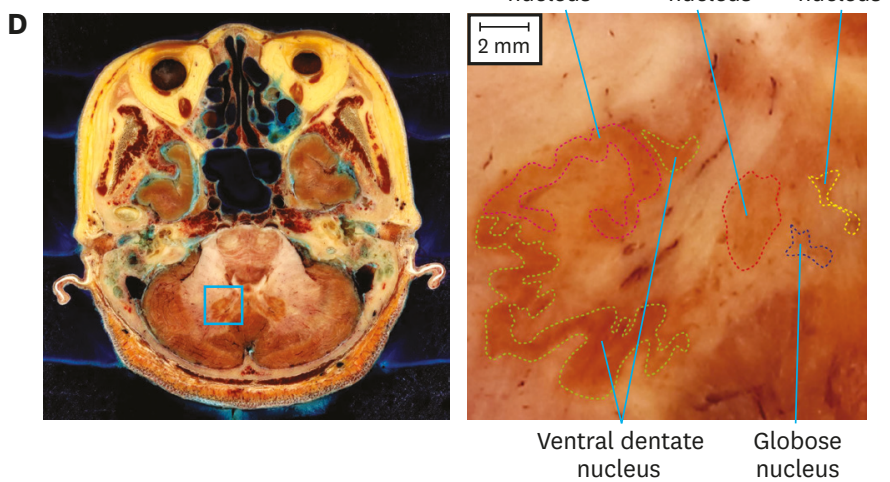

Yücel et al. ${ }^{22}$

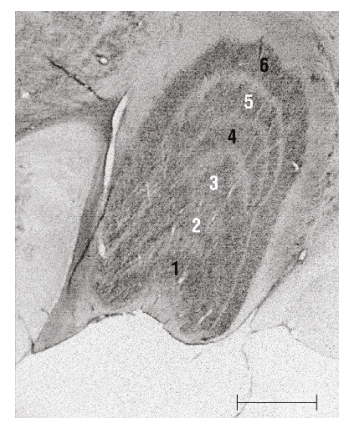

van Dijk et al. ${ }^{23}$

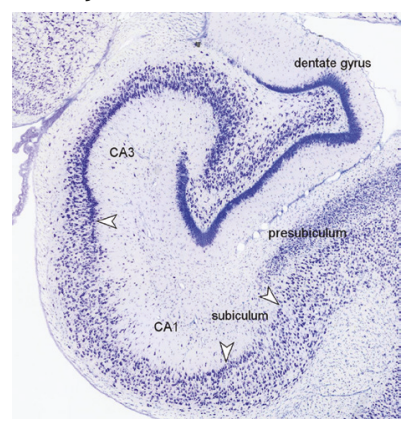

Tellmann et al. ${ }^{24}$

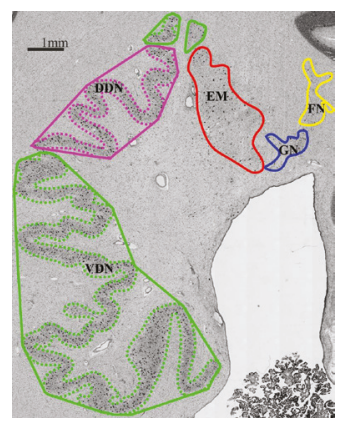

Fig. 3. Sectioned images showing the cerebrum. (A) Thalamus, (B) lateral geniculate nucleus, (C) hippocampus, and (D) deep cerebellar nuclei are identified on the horizontal sectioned images. The area selected with a green box on the overview images (left) is magnified (middle) to be compared with the neurohistological stains of neuroanatomy textbooks (right) at 1st, 2nd, 3rd, and 4th rows.

$\mathrm{CeM}=$ central medial nucleus, $\mathrm{CM}=$ centromedial nucleus, $\mathrm{GPi}=$ globus pallidus internal segment, GPe = globus pallidus external segment, $\mathrm{Hb}=$ habenula, ic = internal capsule, $\mathrm{Li}=$ limitans nucleus, $\mathrm{mtt}=$ mamillothalamic tract, $\mathrm{Pf}=$ parafascicular nucleus, $\mathrm{PuL}=$ lateral pulvinar, $\mathrm{PuM}=\mathrm{medial}$ pulvinar, $\mathrm{VAmc}=$ ventral anterior nucleus magnocellular, VApc $=$ ventral anterior nucleus parvocellular, VLa $=$ ventral lateral anterior nucleus, VL $p=$ ventral lateral posterior nucleus, VPL $=$ ventral posterior lateral nucleus. 


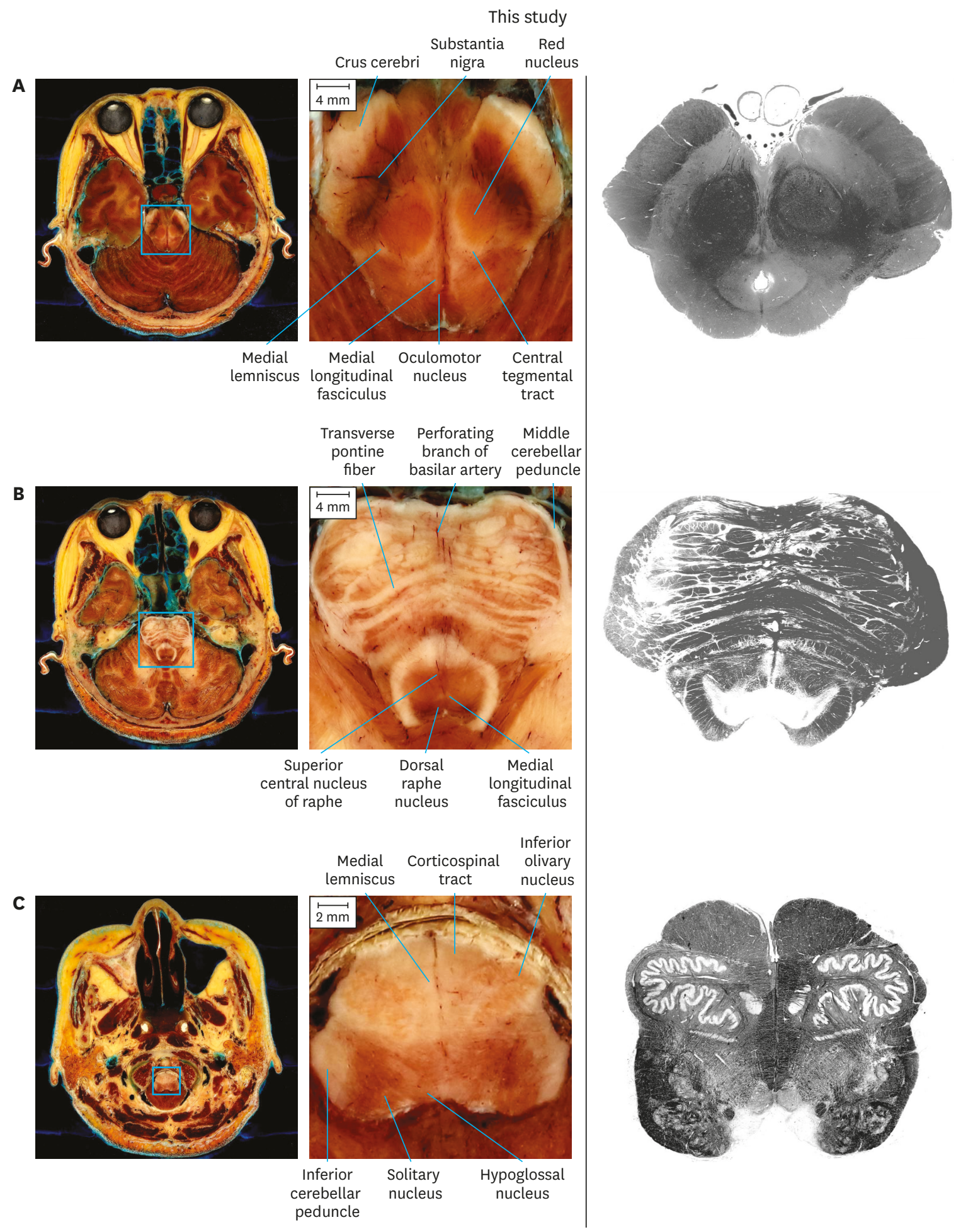

Fig. 4. Sectioned images showing the brainstem. (A) Midbrain, (B) pons, (C) rostral medulla oblongata, and (D) cervical spinal cord identified on the sectioned images. The area selected with a green box on the overview images (left) is magnified (middle) to be compared with the neurohistological stains slides (right). 


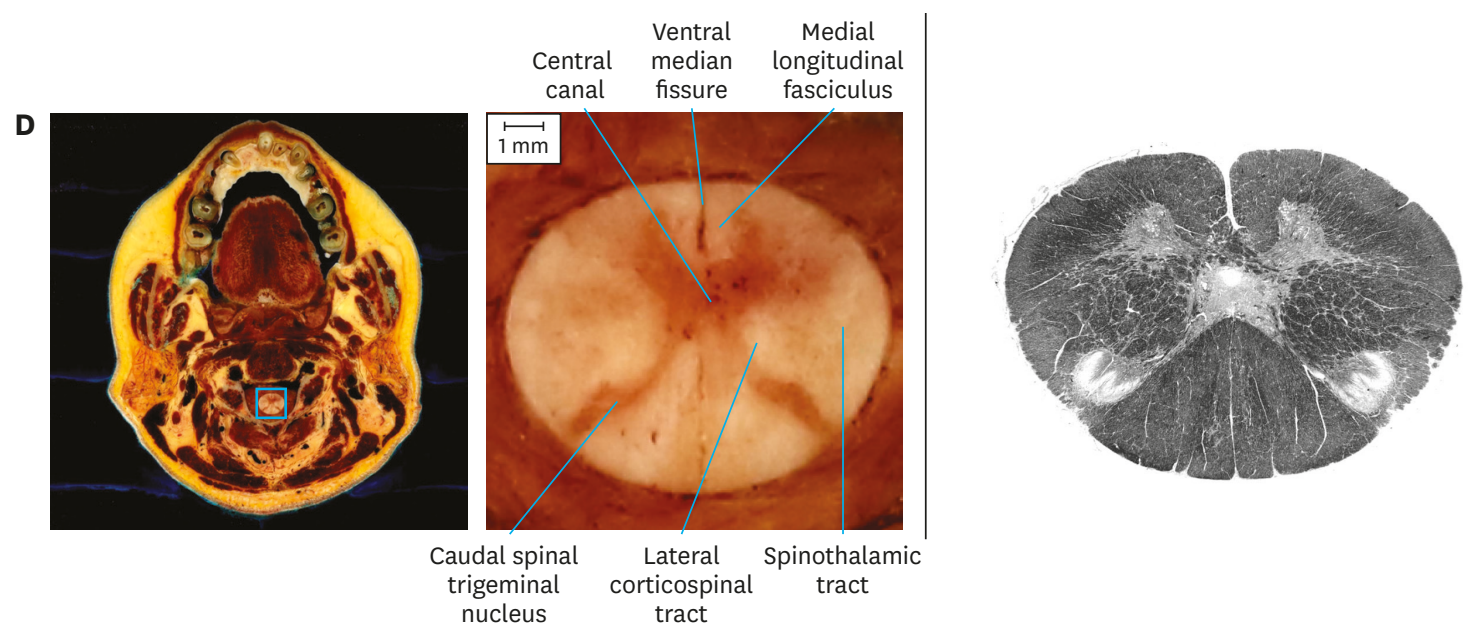

Fig. 4. (Continued) Sectioned images showing the brainstem. (A) Midbrain, (B) pons, (C) rostral medulla oblongata, and (D) cervical spinal cord identified on the sectioned images. The area selected with a green box on the overview images (left) is magnified (middle) to be compared with the neurohistological stains slides (right).
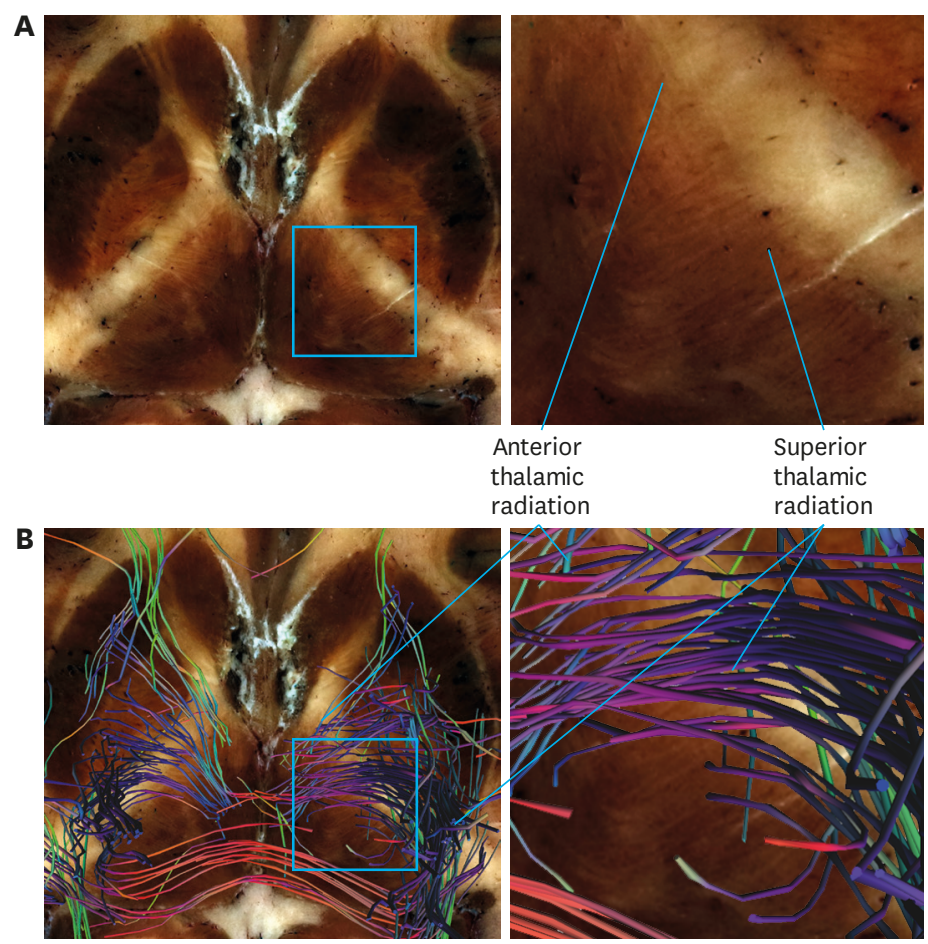

Fig. 5. White matter fibers identified on a sectioned image (A) and a DTI tractrograph of the cadaver (B). Both on a sectioned image and DTI tractrograph, the anterior thalamic radiation shows the fiber direction to the anterior side, while the superior thalamic radiation shows the fiber direction to the lateral side. On the overview of the DTI tractrograph, the destinations of the fibers are indicated (bottom left).

DTI = diffusion tensor image.

\section{DISCUSSION}

As the resolution of MRIs improves continuously, an increasing number of structures can be identified on the MRIs. A more detailed sectional anatomy should be understood to interpret the MRIs precisely. To understand the detailed sectional anatomy, more detailed educational 
resources than the state of the art 7T MRIs is required. Although upcoming technologies, including 14T MRIs, will have an improved resolution, their intrinsic limitation of grayscale and blurriness cannot be overcome. ${ }^{25}$ Consequently, the sectioned images will be an ideal resource for examining the improved MRIs, owing to their higher resolution and actual color.

The image resolution of the our previous study was $4,368 \times 2,912,{ }^{1}$ while that of this study was $8,688 \times 5,792$. Both the horizontal and vertical pixel numbers were increased almost two-fold. To reduce the pixel size of the new images, the field of view was reduced. Moreover, the sectioning interval was reduced to manufacture improved sectioned images of a cadaver head. As a result, the new images have a voxel size of $0.04 \mathrm{~mm} \times 0.04 \mathrm{~mm} \times 0.04 \mathrm{~mm}$, which is $40 \%$ of the previous images in every direction; in other words, 2.5 fold higher resolving power (Fig. 6). ${ }^{25}$

The continuously improving sectioned images of a cadaver head are useful for multiple purposes as follows.

First, the sectioned images can compensate the conventional neurohistological stains. Although the resolution of the sectioned images is less powerful than that of stained specimens, the sectioned images can be useful in the field of neuroanatomy. Stained specimens show only selected structures (e.g., only the nuclei in Nissl staining, and only the myelin sheaths in Luxol-fast blue staining), the sectioned images can show all the visible structures in actual color (Figs. 3 and 4 ).

This study

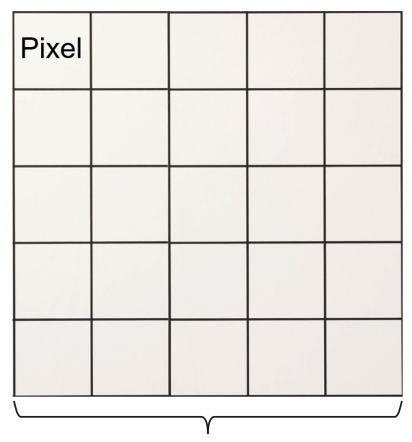

$0.2 \mathrm{~mm}$

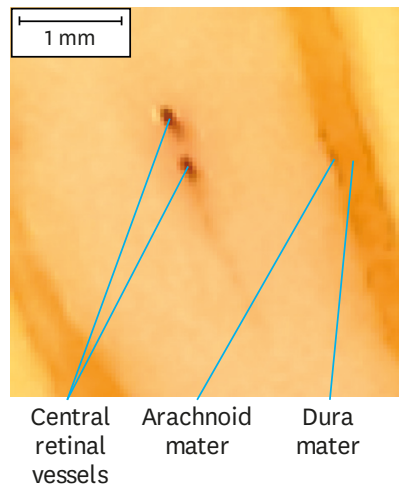

Park et al.

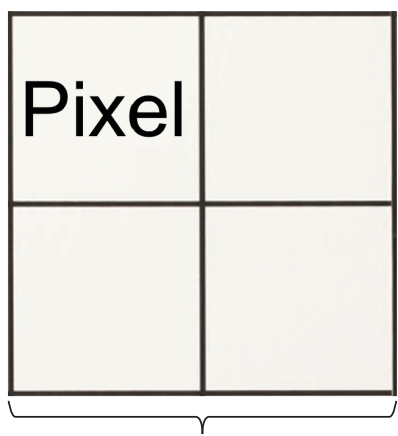

$0.2 \mathrm{~mm}$

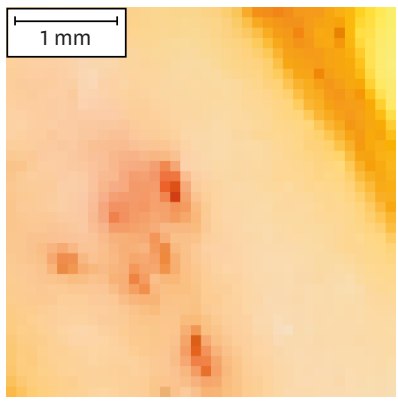

Jones $^{26}$

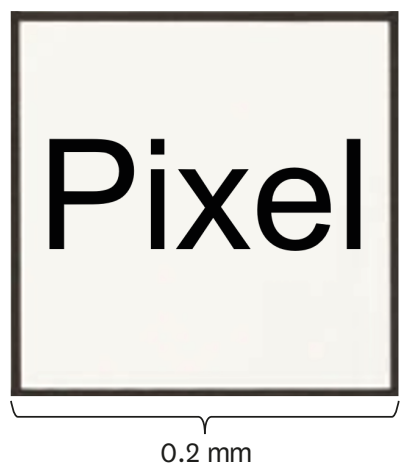

$0.2 \mathrm{~mm}$

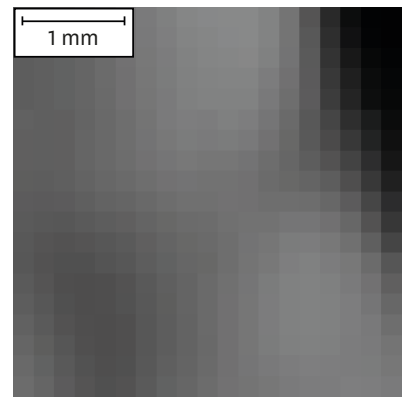

Fig. 6. Pixel sizes of the new sectioned images, previous sectioned images, and 7 Tesla magnetic resonance images (1st row). ${ }^{26}$ As a representative, the vessels and meninges of the optic nerve are displayed (2nd row). The pixelation is least visible on the new sectioned images of this study. 
Second, the sectioned images themselves are useful neuroanatomy content. The previous sectioned images of the head were made into an atlas for sectional anatomy on their own ${ }^{2}$ and along with 7T MRIs. ${ }^{3}$ The new sectioned images with 3T MRIs and DTI of a cadaver have greater potential to depict detailed sectional anatomy. ${ }^{27}$

Third, the new sectioned images can be made into 3D models for educational purposes as in previous research. ${ }^{7111,12}$ The application of 3D printing ${ }^{28,29}$ and augmented reality ${ }^{30}$ will enhance the learning effects of these models.

Fourth, the 3D models can be used for virtual experiments on the effects of electromagnetic waves and radiation, similar to sectioned images. ${ }^{31-33}$

Twenty years earlier, the image resolution of this study was believed to be unachievable. As the resolution of digital cameras improve, the quality of the sectioned images improve. In another 20 years, it is difficult to estimate how the resolution of sectioned images will improve. Therefore, the ultimate aim of sectioned images is to reach the field of microscopic observations. The sectioned images of this study will serve as foundation material in the field of sectional anatomy. To other researchers to produce a virtual experiment and virtual reality using 3D models, the images of this study will provided free of charge in anatomy.co.kr.

\section{REFERENCES}

1. Park JS, Chung MS, Shin DS, Har DH, Cho ZH, Kim YB, et al. Sectioned images of the cadaver head including the brain and correspondences with ultrahigh field 7.0 T MRIs. Proc IEEE 2009;97(12):1988-96. CROSSREF

2. Park JS. Cross-Sectional Atlas of the Human Head: with 0.1-mm Pixel Size Color Images. Berlin: Springer; 2018.

3. Cho ZH. 7.0 Tesla MRI Brain Atlas: In Vivo Atlas with Cryomacrotome Correlation. Berlin: Springer Science \& Business Media; 2010.

4. Park JS, Jung YW. Software for browsing sectioned images of a dog body and generating a 3D model. Anat Rec (Hoboken) 2016;299(1):81-7. PUBMED | CROSSREF

5. Kwon K, Chung MS, Park JS, Shin BS, Chung BS. Improved software to browse the serial medical images for learning. J Korean Med Sci 2017;32(7):1195-201. PUBMED | CROSSREF

6. Lee SB, Chung BS, Chung MS, Youn C, Park JS. Browsing software of the head sectioned images for the android mobile device. Int J Morphol 2017;35(4):1377-82. CROSSREF

7. Shin DS, Jang HG, Park JS, Park HS, Lee S, Chung MS. Accessible and informative sectioned images and surface models of a cadaver head. J Craniofac Surg 2012;23(4):1176-80. PUBMED | CROSSREF

8. Park HS, Chung MS, Shin DS, Jung YW, Park JS. Accessible and informative sectioned images, color-coded images, and surface models of the ear. Anat Rec (Hoboken) 2013;296(8):1180-6. PUBMED | CROSSREF

9. Park HS, Chung MS, Shin DS, Jung YW, Park JS. Whole courses of the oculomotor, trochlear, and abducens nerves, identified in sectioned images and surface models. Anat Rec (Hoboken) 2015;298(2):436-43. PUBMED | CROSSREF

10. Chung BS, Ahn YH, Park JS. Ten triangles around cavernous sinus for surgical approach described by schematic diagram and three dimensional models with the sectioned images. J Korean Med Sci 2016;31(9):1455-63. PUBMED | CROSSREF

11. Chung BS, Shin DS, Brown P, Choi J, Chung MS. Virtual dissection table including the visible Korean images complemented by free software of the same data. Int J Morphol 2015;33(2):440-5. CROSSREF 
12. Chung BS, Park JS. Real-color volume models made from real-color sectioned images of visible Korean. J Korean Med Sci 2019;34(10):e86. PUBMED | CROSSREF

13. Morel A. Stereotactic Atlas of the Human Thalamus and Basal Ganglia. Boca Raton FL, CRC Press; 2007.

14. Waldvogel HJ, Curtis MA, Baer K, Rees MI, Faull RL. Immunohistochemical staining of post-mortem adult human brain sections. Nat Protoc 2006;1(6):2719-32. PUBMED | CROSSREF

15. Friedland DR, Los JG, Ryugo DK. A modified Golgi staining protocol for use in the human brain stem and cerebellum. J Neurosci Methods 2006;150(1):90-5. PUBMED | CROSSREF

16. Bürgel U, Amunts K, Hoemke L, Mohlberg H, Gilsbach JM, Zilles K. White matter fiber tracts of the human brain: three-dimensional mapping at microscopic resolution, topography and intersubject variability. Neuroimage 2006;29(4):1092-105. PUBMED | CROSSREF

17. Tamraz JC, Comair YG, Tamraz JC. Atlas of Regional Anatomy of the Brain Using MRI. Springer-Verlag; 2004.

18. Tzourio-Mazoyer N, Landeau B, Papathanassiou D, Crivello F, Etard O, Delcroix N, et al. Automated anatomical labeling of activations in SPM using a macroscopic anatomical parcellation of the MNI MRI single-subject brain. Neuroimage 2002;15(1):273-89.

PUBMED | CROSSREF

19. Park JS, Chung MS, Park HS, Shin DS, Har DH, Cho ZH, et al. A proposal of new reference system for the standard axial, sagittal, coronal planes of brain based on the serially-sectioned images. J Korean Med Sci 2010;25(1):135-41. PUBMED | CROSSREF

20. Li X, Morgan PS, Ashburner J, Smith J, Rorden C. The first step for neuroimaging data analysis: DICOM to NIfTI conversion. J Neurosci Methods 2016;264:47-56. PUBMED | CROSSREF

21. Niemann K, Mennicken VR, Jeanmonod D, Morel A. The Morel stereotactic atlas of the human thalamus: atlas-to-MR registration of internally consistent canonical model. Neuroimage 2000;12(6):601-16. PUBMED | CROSSREF

22. Yücel YH, Zhang Q, Gupta N, Kaufman PL, Weinreb RN. Loss of neurons in magnocellular and parvocellular layers of the lateral geniculate nucleus in glaucoma. Arch Ophthalmol 2000;118(3):378-84. PUBMED | CROSSREF

23. van Dijk RM, Huang SH, Slomianka L, Amrein I. Taxonomic separation of hippocampal networks: principal cell populations and adult neurogenesis. Front Neuroanat 2016;10:22. PUBMED | CROSSREF

24. Tellmann S, Bludau S, Eickhoff S, Mohlberg H, Minnerop M, Amunts K. Cytoarchitectonic mapping of the human brain cerebellar nuclei in stereotaxic space and delineation of their co-activation patterns. Front Neuroanat 2015;9:54. PUBMED | CROSSREF

25. Cao Z, Park J, Cho ZH, Collins CM. Numerical evaluation of image homogeneity, signal-to-noise ratio, and specific absorption rate for human brain imaging at 1.5, 3, 7, 10.5, and $14 \mathrm{~T}$ in an 8-channel transmit/ receive array. J Magn Reson Imaging 2015;41(5):1432-9. PUBMED | CROSSREF

26. Jones SE. Brain lesion conspicuity at 7T: defining the diagnostic value of high-field-strength MRI. https:// consultqd.clevelandclinic.org/brain-lesion-conspicuity-7t-defining-diagnostic-value-high-field-strength/. Updated 2016. Accessed June 14, 2019.

27. Cho ZH. Review of recent advancement of ultra high field magnetic resonance imaging: from anatomy to tractography. Investig Magn Reson Imaging 2016;20(3):141-51. CROSSREF

28. AbouHashem Y, Dayal M, Savanah S, Štrkalj G. The application of 3D printing in anatomy education. Med Educ Online 2015;20(1):29847. PUBMED | CROSSREF

29. Lee S, Kim T, Hong D, Ock J, Kwon J, Gwon E, et al. A review of three-dimensional printing technology for medical applications. J Korean Soc Radiol 2019;80(2):213-25. CROSSREF

30. Thomas RG, John NW, Delieu JM. Augmented reality for anatomical education. J Vis Commun Med 2010;33(1):6-15.

PUBMED | CROSSREF 
31. Kim CH, Jeong JH, Bolch WE, Cho KW, Hwang SB. A polygon-surface reference Korean male phantom (PSRK-Man) and its direct implementation in Geant4 Monte Carlo simulation. Phys Med Biol 2011;56(10):3137-61. PUBMED | CROSSREF

32. Neufeld E, Lloyd B, Schneider B, Kainz W, Kuster N. Functionalized anatomical models for computational life sciences. Front Physiol 2018;9:1594.

PUBMED | CROSSREF

33. Kim SK, Kim C, Lee KY, Cha J, Lim HJ, Kang EY, et al. Accuracy of model-based iterative reconstruction for CT Volumetry of part-solid nodules and solid nodules in comparison with filtered back projection and hybrid iterative reconstruction at various dose settings: an anthropomorphic chest phantom study. Korean J Radiol 2019;20(7):1195-206.

PUBMED | CROSSREF 